\section{Ziprasidone-Induced Angioedema: A Case Report}

Sir: Ziprasidone is an antipsychotic agent that is generally well tolerated. Allergic responses to ziprasidone have been reported, including immunoglobulin E (IgE)-related pedal edema, ${ }^{1}$ lifethreatening hypersensitivity syndrome, ${ }^{2}$ and angioedema. ${ }^{3}$ We present a report of a patient who developed angioedema upon starting ziprasidone treatment.

Case report. Mr A is a 30-year-old man who presented with psychotic symptoms characterized by persecutory delusions over a 6-week period. His diagnosis according to DSM-IV-TR was bipolar I disorder, most recent episode depressed, severe with psychotic features. He had had 2 previous episodes of mania with psychosis that were managed with short-term antipsychotic medication (olanzapine for the first episode and aripiprazole for the second) and maintenance mood stabilizers (lithium and sodium valproate for the second episode) without any adverse effects.

Mr A's treating psychiatrist started him on ziprasidone therapy without any other concurrent medication. Prior to treatment with ziprasidone, $\mathrm{Mr}$ A had been free of all other prescription and nonprescription medication in the preceding 3 months. He commenced ziprasidone $20 \mathrm{mg}$ twice daily on the first day and had a 40-mg tablet the next morning. Within 6 hours of taking the 40-mg dose, his tongue began to swell and he could not speak clearly. He experienced shortness of breath and was immediately transferred to the hospital by ambulance. Mr A had no past history of asthma, allergic rhinitis, urticaria, food allergies, eczematous dermatitis, or drug-related allergies.

On examination, his tongue and lips were swollen, extending up to the jaw line. There was partial obstruction to the airway, and the patient found it difficult to breathe through the mouth. There was no swelling noted of the genitalia, palms, soles, or eyelids. He had tachycardia, mildly elevated blood pressure, and an oxygen saturation of $97 \%$. There was no lymphadenopathy or urticarial lesions, and the findings from systemic examination were normal.

The diagnostic impression was that Mr A's presentation possibly represented an anaphylactic reaction to ziprasidone. He was immediately treated with nebulized and intramuscular epinephrine, intravenous hydrocortisone, and promethazine. He responded to these interventions, and within 10 minutes, he was able to talk and breathe without distress. Within 4 hours, the swelling subsided completely, and he could tolerate oral fluids. Apart from a marginal increase in white cell count, the results of blood investigations were otherwise normal. Mr A was discharged from the medical unit after 48 hours and transferred to the psychiatric ward. The medical team advised the patient to continue oral prednisolone for 5 days.

The adverse reaction scored 6 on Naranjo and colleagues' Adverse Drug Reaction (ADR) scale, ${ }^{4}$ thus implicating ziprasidone as a probable cause for the event. While in the psychiatric unit, Mr A was commenced on amisulpride treatment after a 5-day medication-free period. There was no recurrence of angioedema with amisulpride. His psychotic symptoms improved within a week, and there were no further allergic reactions in the subsequent 3 months.

Angioedema is a well-demarcated localized edema involving the deeper layers of the skin, including the subcutaneous tissue. It may occur as an IgE-dependent allergic reaction to a specific antigen such as drugs, although other immunologic mechanisms are postulated. Angioedema of the upper respiratory tract may be lifethreatening due to laryngeal obstruction. ${ }^{5}$ The clinical picture of $\mathrm{Mr} \mathrm{A}$ and temporal association with ziprasidone intake suggest a direct potentially life-threatening immunologic response to ziprasidone. A limitation of our report is the absence of an intradermal skin test that could have conclusively implicated ziprasidone as the etiologic allergen. Risperidone ${ }^{6}$ and clozapine $e^{7}$ are other antipsychotic agents reported in the literature to elicit angioedema.

This is the second case report about angioedema occurring after ziprasidone intake. Clinicians in their psychoeducation with patients should discuss the possibility of severe allergic reactions when commencing ziprasidone treatment, which may ensure early recognition and intervention of potentially life-threatening angioedema.

\section{ReFEREnCES}

1. Ku HL, Su TP, Chow YH. Ziprasidone-associated pedal edema in the treatment of schizophrenia. Prog Neuropsychopharmacol Biol Psychiatry. 2006;30(5):963-964.

2. Tsai CF, Tsai SJ, Hwang JP. Ziprasidone-induced hypersensitivity syndrome in an aged schizophrenia patient [letter]. Int J Geriatr Psychiatry. 2005;20(8):797-799.

3. Akkaya C, Sarandol A, Aydogan K, et al. Urticaria and angio-oedema due to ziprasidone. J Psychopharmacol. 2007;21(5):550-552.

4. Naranjo CA, Busto U, Sellers EM. A method for estimating the probability of adverse drug reactions. Clin Pharmacol Ther. 1981;30:239-245.

5. Austen KF. Allergies, anaphylaxis and systemic mastocytosis. In: Fauci AS, Braunwald E, Kasper DL, et al, eds. Harrison's Principles of Internal Medicine, vol. 2. 17th ed. New York, NY: McGraw Hill; 2008:2061-2070.

6. Cooney C, Nagy A. Angio-oedema associated with risperidone [letter]. BMJ. 1995;311(7014):1204.

7. Mishra B, Sahoo S, Sarkar S. Clozapine-induced angioneurotic edema. Gen Hosp Psychiatry. 2007;29:78-80.

Titus Mohan, MD

Tarun Bastiampillai, FRANZCP Rohan Dhillon, FRANZCP

Author affiliations: The Queen Elizabeth Hospital, South Australia, Australia. Financial disclosure: None reported. Funding/support: None reported. doi:10.4088/JCP.08104657

(c) Copyright 2009 Physicians Postgraduate Press, Inc.

\section{Effects of Bupropion Augmentation in Escitalopram-Resistant Patients With Major Depressive Disorder: An Open-Label, Naturalistic Study}

Sir: There is a pressing need for improvement of treatment response to antidepressants in depression. Increasing evidence shows that bupropion, a norepinephrine and dopamine reuptake inhibitor, is one of the most widely chosen and effective augmenting agents for depressive patients with insufficient response to serotonin reuptake inhibitors. ${ }^{1-3}$ In this study, we evaluated the efficacy and tolerability of bupropion augmentation in nonresponders to escitalopram monotherapy in order to better understand this potential approach to improving treatment outcome in depression.

Method. The study sample consisted of 135 subjects with major depressive disorder (mean \pm SD age $=31.1 \pm 11.6$ years, $66.1 \%$ female) recruited from outpatients admitted to the Psychiatry Clinic of the Tartu University Hospital in Tartu, Estonia, between December 2006 and March 2008. Diagnosis according to DSM-IV criteria was determined using the Mini-International Neuropsychiatric Interview (MINI, version 5.0.0) ${ }^{4}$ and substantiated 
by psychiatric history and medical records. A depression severity of at least "moderate" was required for inclusion as indicated by a Montgomery-Asberg Depression Rating Scale (MADRS) ${ }^{5}$ total score of 22 or higher. Only secondary current comorbid anxiety disorders, including generalized anxiety disorder and social phobia, but not other psychiatric or somatic diseases were allowed. None of the patients had psychotic features during their depressive episodes or met criteria for psychotic depression. Patients were treated with escitalopram 10-20 mg/day for 12 weeks in an openlabel naturalistic design. No other medications, except zolpidem or zopiclone for insomnia, were allowed during the study. After 12 weeks, the nonresponders to $20 \mathrm{mg}$ of escitalopram monotherapy were given a combination of $20 \mathrm{mg}$ of escitalopram and 150-300 $\mathrm{mg}$ /day of bupropion for an additional 6 weeks. Clinical severity and treatment response were assessed biweekly using the MADRS and Clinical Global Impressions (CGI) scale. ${ }^{6}$ The 17 -item Hamilton Rating Scale for Depression (HAM-D-17) ${ }^{7}$ and the Beck Depression Inventory (BDI $)^{8}$ were also used as secondary assessments of depressive symptoms, and adverse effects were reported on the Toronto Side Effect Scale (TSES) ${ }^{9}$ at each visit. There were high correlations between assessments on the clinical scales MADRS or HAM-D-17 and the BDI self-evaluations.

At week 4 of the initial 12-week study, the dose of escitalopram was increased and kept at $20 \mathrm{mg} /$ day until the end of the study in patients who demonstrated less than a 50\% decrease in MADRS total score. Patients showing at least 50\% decline in the MADRS total score at week 4 continued on the 10 -mg dose. However, later the dose was increased and kept at $20 \mathrm{mg}$ in 2 patients who showed exacerbation of depressive symptoms in follow-up visits, one at week 5 and one at 6 .

Bupropion was started at $150 \mathrm{mg} /$ day in the morning and was allowed to increase up to $300 \mathrm{mg} /$ day, given as $150 \mathrm{mg}$ twice daily, after week 2 or later if patients still demonstrated insufficient response according to clinical assessments. The patients were defined as responders if the decrease in both MADRS and HAM-D-17 total scores was at least $50 \%$ and the score on the CGI-Improvement scale was 2 or less. The remitters were defined as those whose scores were less than 12 on the MADRS and less than 8 on the HAM-D-17. The Human Studies Ethics Committee of the University of Tartu approved the study protocol, and all patients provided written informed consent.

Results. At the end of week 12 of treatment with escitalopram, 82 patients $(60.7 \%)$ were defined as responders (Figure 1 ) and 79 of them (58.5\%) achieved remission. Forty-four patients (32.6\%) showed insufficient or partial response to treatment, and 9 patients $(6.7 \%)$ discontinued escitalopram treatment due to lack of efficacy or adverse effects. The daily dose of escitalopram was increased and kept at $20 \mathrm{mg}$ in 85 patients, 41 of whom were responders. The nonresponders to escitalopram monotherapy had significantly higher prevalence of melancholic type of depression than did responders $(86.4 \%$ vs. $63.4 \%$ respectively, $\mathrm{p}=.007)$ and experienced more adverse events, including weakness and fatigue, during the escitalopram stage of the trial according to TSES assessments $(\mathrm{p}<.05)$.

In total, 41 patients showing nonresponse to 8 weeks of monotherapy with $20 \mathrm{mg}$ of escitalopram received bupropion augmentation, whereas 3 patients declined to continue participation in the study due to personal reasons. At week 6 of augmentation, $25(61.0 \%)$ were defined as responders and 22 of them $(53.7 \%)$ achieved remission, whereas 13 patients (31.7\%) showed insufficient or partial response and 3 patients $(7.3 \%)$ discontinued due to adverse effects or lack of efficacy. Bupropion dose was increased to $300 \mathrm{mg}$ in 24 patients, of whom 14 were responders. Only muscle twitching was reported more often or more severely on the TSES in the nonresponders to bupropion augmentation as compared with responders $(\mathrm{p}<.01)$. Moreover, the mean \pm SD daily dose
Figure 1. Mean Change in Montgomery-Asberg Depression Rating Scale (MADRS) Scores for Responders and Nonresponders to Escitalopram Monotherapy and Bupropion Augmentation ${ }^{\mathrm{a}}$

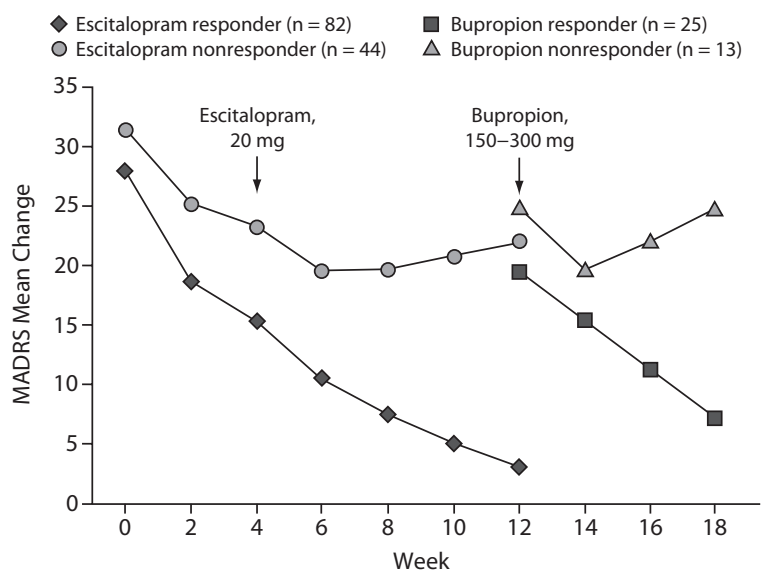

aAt baseline, the severity of depression on the MADRS scale was significantly lower in responders to escitalopram monotherapy as compared with nonresponders $(\mathrm{t}=19.79, \mathrm{df}=55,80 ; \mathrm{p}<.0001)$. The responders to bupropion augmentation had significantly lower severity of depression on the MADRS scale before starting of augmentation (week 12) than nonresponders $(\mathrm{t}=2.60 ; \mathrm{df}=21,0 ; \mathrm{p}=.01)$.

of bupropion did not significantly differ between the 2 groups ( $265 \pm 66 \mathrm{mg}$ vs. $234 \pm 76 \mathrm{mg}$, respectively, $\mathrm{p}=.30$ ), demonstrating also that frequency or severity of adverse effects was not related to administered dose of medication. None of the following characteristics differed significantly between responders and nonresponders to bupropion augmentation: age, sex, onset of disease, melancholic features, number of previous depressive episodes, and duration of current depressive episode.

In agreement with previous studies, ${ }^{10-12}$ we found that bupropion augmentation successfully facilitated treatment response in nonresponders to monotherapy with an SSRI. However, our remission rate with bupropion augmentation was higher than those demonstrated by the Sequenced Treatment Alternatives to Relieve Depression (STAR*D) trial, ${ }^{11}$ probably due to lower dropout and discontinuation rates and to methodological differences. We found that the melancholic features of depression were associated with insufficient or partial response to escitalopram and that these could effectively be resolved by bupropion augmentation. Our results may give additional support to the importance of focusing treatment on the predominant or driving symptomatology of depression in order to maximize the chances of response and remission among patients and suggest that the use of bupropion is an appropriate treatment for patients with melancholic type of depression. ${ }^{13-15}$ Importantly, there were similarly low proportions of patients who discontinued either monotherapy with escitalopram or combined treatment with bupropion due to adverse events, indicating that both medications were generally well tolerated. Although our response and remission rates with escitalopram monotherapy were comparable to those reported by previous randomized and controlled clinical trials with escitalopram, ${ }^{16}$ we showed that almost all responders fulfilled the criteria for remission. We suggest that a longer treatment period and relatively earlier increasing of dose up to $20 \mathrm{mg} /$ day (from week 4) might significantly reduce the difference between the response and remission rates, due to cumulative treatment efficacy in some patients. In addition, a consistent 
increase in response rates was also demonstrated in another study using long-term treatment with escitalopram, ${ }^{17}$ suggesting that responders are more likely to achieve remission during longer treatment periods. Although severity of depressive symptoms and treatment response were carefully rated at each visit and were supported by patients' self-evaluation, placebo responses cannot be excluded in our study due to the naturalistic, open-label design. Further randomized clinical trials with longer follow-up periods and larger sample sizes would be necessary to evaluate the efficacy of bupropion augmentation in resistant depression and particularly with melancholic features.

This investigation was supported by Estonian Science Foundation grant 7034 (Dr. Maron) and target grant SF0180125s08 (Dr. Vasar) from the Ministry of Education of Estonia.

Drs. Maron and Nutt have served as consultants for and have received grants and honoraria from Lundbeck and GlaxoSmithKline. Drs. Eller and Vasar report no additional financial or other relationships relevant to the subject of this letter.

Trial Registration: www.anzctr.org.au Identifier ACTRN12609000295246.

\section{REFERENCES}

1. Mischoulon D, Nierenberg AA, Kizilbash L, et al. Strategies for managing depression refractory to selective serotonin reuptake inhibitor treatment: a survey of clinicians. Can J Psychiatry 2000;45(5):476-481

2. Zisook S, Rush AJ, Haight BR, et al. Use of bupropion in combination with serotonin reuptake inhibitors. Biol Psychiatry 2006;59(3):203-210

3. Stahl SM, Pradko JF, Haight BR, et al. A review of the neuropharmacology of bupropion, a dual norepinephrine and dopamine reuptake inhibitor. Prim Care Companion J Clin Psychiatry 2004;6(4):159-166

4. Sheehan DV, Lecrubier Y, Sheehan KH, et al. Mini-International Neuropsychiatric Interview (M.I.N.I.): the development and validation of a structured diagnostic psychiatric interview for DSM-IV and ICD-10. J Clin Psychiatry 1998;59(suppl 20):22-33

5. Montgomery SA, Asberg MC. A new depression rating scale designed to be sensitive to change. Br J Psychiatry 1979;134:382-389

6. Guy W. EDCEU Assessment Manual for Psychopharmacology. US Dept Health, Education, and Welfare publication (ADM) 76-338. Rockville, MD: National Institute of Mental Health; 1976;218-222

7. Hamilton M. A rating scale for depression. J Neurol Neurosurg Psychiatry 1960;23:56-62

8. Beck AT, Ward CH, Mendelson M, et al. An inventory for measuring depression. Arch Gen Psychiatry 1961;4:561-571

9. Vanderkooy JD, Kennedy SH, Bagby RM. Antidepressant side effects in depression patients treated in a naturalistic setting: a study of bupropion, moclobemide, paroxetine, sertraline, and venlafaxine. Can J Psychiatry 2002;47(2):174-180

10. Papakostas GI, Worthington JJ 3rd, Iosifescu DV, et al. The combination of duloxetine and bupropion for treatment-resistant major depressive disorder. Depress Anxiety 2006;23(3):178-181

11. Trivedi MH, Fava M, Wisniewski SR, et al. Medication augmentation after the failure of SSRIs for depression. N Engl J Med 2006;354(12):1243-1252

12. Lam RW, Hossie H, Solomons K, et al. Citalopram and bupropion-SR: combining versus switching in patients with treatment-resistant depression. J Clin Psychiatry 2004;65(3):337-340

13. Jefferson JW, Rush AJ, Nelson JC, et al. Extended-release bupropion for patients with major depressive disorder presenting with symptoms of reduced energy, pleasure, and interest: findings from a randomized, double-blind, placebo-controlled study. J Clin Psychiatry 2006;67(6):865-873

14. Papakostas GI, Nutt DJ, Hallett LA, et al. Resolution of sleepiness and fatigue in major depressive disorder: a comparison of bupropion and the selective serotonin reuptake inhibitors. Biol Psychiatry 2006;60(12):1350-1355

15. Nutt DJ, Demyttenaere K, Janka Z, et al. The other face of depression, reduced positive affect: the role of catecholamines in causation and cure. J Psychopharmacol 2007;21(5):461-471

16. Höschl C, Svestka J. Escitalopram for the treatment of major depression and anxiety disorders. Expert Rev Neurother 2008;8(4):537-552

17. Wade A, Despiegel N, Heldbo Reines E. Escitalopram in the longterm treatment of major depressive disorder. Ann Clin Psychiatry 2006;18(2):83-89

Eduard Maron, M.D., Ph.D. Research Department of Mental Health North Estonia Medical Centre Foundation Psychiatry Clinic Tallinn, Estonia Department of Psychiatry University of Tartu Tartu, Estonia

Triin Eller, M.D.

Veiko Vasar, M.D.

Department of Psychiatry University of Tartu Tartu, Estonia

David J. Nutt, D.M., F.R.C.Psych. Department of Community Based Medicine Psychopharmacology Unit University of Bristol Bristol, United Kingdom

doi:10.4088/JCP.08104477 (c) Copyright 2009 Physicians Postgraduate Press, Inc.

\section{Pseudohallucinations Versus True Hallucinations in Prodromal Psychosis: Does It Really Matter?}

Sir: According to the traditional accounts of European psychiatry, true hallucinations are apparent perceptions of an external object in the absence of adequate sensory stimuli. Conversely, Sims ${ }^{1}$ states that Kandinsky and Jaspers described pseudohallucinations as a separate form of perception from true hallucination. Pseudohallucination is a perceptual experience that is figurative, not concrete or "real," is located in inner subjective space, and is perceived with the "inner" ear (or eye) (Table 1). In other characteristics, pseudohallucinations are more like true hallucinations than fantasy. Thus, pseudohallucination may have definite outline and vivid detail, it may be retained for some time, and it is not deliberately evoked. ${ }^{3}$ Jaspers $^{2}$ stressed that there is a gradation from the more fully formed pseudohallucination to vivid imagery but that there is an absolute distinction between hallucination and pseudohallucination because of the inner location of the latter. As a consequence of these original speculations, it is a common belief that pseudohallucinations do not have the same psychiatric significance as true hallucinations, and thus clinicians expend some clinical effort to distinguish the two. True hallucinations are thought to be always indicative of a morbid mental state such as psychosis, while pseudohallucinations are thought to be of less diagnostic significance and not necessarily psychopathological. However, pseudohallucinations may be an attenuated and subtle expression of an evolving psychosis. There have been no previous studies of the degree to which pseudohallucinations are predictive of the subsequent development of psychosis.

Here we report 5 cases of young (age range, 18-26 years), drugnaive subjects presenting at a clinical service for prodromal signs of psychosis ${ }^{4}$ because they were hearing internal voices (pseudohallucinations). At the time of the first assessment (2005 through 2008), these symptoms did not meet DSM-IV threshold for a psychotic episode but met the inclusion clinical criteria for an "at-risk mental state" (ARMS), which is associated with an elevated clinical risk of psychosis. ${ }^{5}$ The diagnosis was based on assessment by 2 experienced clinicians using the Comprehensive Assessment for the ARMS (CAARMS). ${ }^{4,5}$ 


\begin{tabular}{llll}
\hline \multicolumn{4}{l}{ Table 1. Psychopathological Features of Hallucination, Pseudohallucination, and Imagery } \\
\hline Domain & \multicolumn{1}{c}{ Hallucination } & \multicolumn{1}{c}{ Pseudohallucination } \\
\hline 1. Experience & Concrete, tangible, objective, real & Pictorial subjective & Imagery \\
2. Location & Outer objective space & Inner subjective space & Pictorial subjective \\
3. Definition & Definite outlines, complete sound & Definite outlines, complete sound & Inner subjective space \\
4. Vividness & Full, fresh, bright & Full, fresh, bright & Most elements are dim on neutral \\
5. Constancy & Retained & Retained & Evanescent \\
6. Independence & Cannot be dismissed, recalled, or & Cannot be dismissed, recalled, or & Requires voluntary creation \\
from volition & changed at will & changed at will & \\
\hline
\end{tabular}

Partly derived from information in Jaspers. ${ }^{2}$

Case 1. Mr. A was an 18-year-old man who experienced auditory perception abnormalities in that he heard internal voices in the third person commenting on his actions. They typically happened a few times per week and each episode lasted only 5-10 minutes. He recognized that the voices were similar to those of friends, teachers, or his dad and could be neutral or critical in their content, although occasionally they offered helpful advice.

Case 2. Mr. B was a 26-year-old man who reported a voice he heard inside his head on a weekly basis, which was triggered by stressful events such as attending college. He was unable to discriminate it from his own thoughts, although it sounded like a young man's voice talking to him in the second person, commenting on his actions and suggesting that he confront people around him.

Case 3. Mr. C was a 20-year-old man who could hear his thoughts aloud in his mind, although he had no awareness of speaking himself. He felt that the voice was in his head, and it sounded the way he would sound normally, but he did not have any control over it and was unable to distract himself. He added that these thoughts in his head became amplified and sometimes drowned out everything else happening around him.

Case 4. Mr. D was a 20-year-old man who reported hearing a voice inside his head that was distinct from his normal thoughts in that its content was repetitive. The voice would repeat a few statements for more than 1 hour at times. He did not recognize the voice and did not have any theories about the cause of the experience but reported that it "sounded" like a male voice.

Case 5. Ms. E was a 21-year-old woman suffering from an alien (male) voice. Although she could hear it inside her head (as opposed to being an external sound), it was vivid and clear. The voice, which occurred almost daily and correlated with her levels of stress, expressed thoughts that she recognized as her own, and it was difficult for her to distinguish them from her thoughts. She found the voice distressing but was perfectly capable of not doing what the voice requested by keeping very busy and listening to music.

These subjects were followed up by the prodromal team, and, over the following months, they all developed a psychotic episode and were referred to the local first episode service. Transition was defined as the onset of frank psychotic symptoms (above the psychotic threshold on the CAARMS) that did not resolve within a week. $^{5}$

Albeit no quantitative data are available, the present case series strongly suggests that pseudohallucinations may clinically characterize an impending risk for psychosis. Their presence should not be easily dismissed when assessing young subjects seeking help for prodromal symptoms of schizophrenia but should be carefully investigated through a clinical assessment and accurately monitored during an assertive follow-up.

The authors report no financial or other relationships relevant to the subject of this letter.

\section{REFERENCES}

1. Sims A. Symptoms in the Mind: An Introduction to Descriptive Psychopathology. 3rd ed. London: WB Saunders Co; 2003:108-111. 2. Jaspers K. General Psychopathology. Vol. 1. Hoenig J, Hamilton MW, trans. Baltimore, Md: Johns Hopkins University Press; 1997

3. Taylor FK. On pseudohallucinations. Psychol Med 1981;11(2):265-271

4. Broome MR, Woolley JB, Johns LC, et al. Outreach and support in south London (OASIS): implementation of a clinical service for prodromal psychosis and the at-risk mental state. Eur Psychiatry 2005;20(5-6):372-378

5. Yung AR, Phillips LJ, Yuen HP, et al. Psychosis prediction: 12-month follow-up of a high-risk ("prodromal") group. Schizophr Res 2003;60(1):21-32

Paolo Fusar-Poli, M.D., Ph.D. Oliver D. Howes, M.R.C.Psych., D.M. Philip McGuire, M.D., Ph.D., F.R.C.Psych. Department of Psychological Medicine Institute of Psychiatry London and the OASIS team National Health Service Lambeth Trust London, England

(C) Copyright 2009 Physicians Postgraduate Press, Inc.

\section{A 5-Year Follow-Up of Diabetes Knowledge in Persons With Serious Mental Illness and Type 2 Diabetes}

Sir: Despite the high prevalence of type 2 diabetes in persons with serious mental illness, there has been only limited study of diabetes knowledge in this group. In a previous report, we found that among 201 persons with serious mental illness and type 2 diabetes, the mean score on a diabetes knowledge test was only $54 \% .{ }^{1}$ We reassessed the diabetes knowledge of persons in our sample 5 years later, in a period of heightened focus on diabetes among persons with serious mental illness.

Method. We recruited psychiatric outpatients with type 2 diabetes and either schizophrenia or major mood disorder as previously described. ${ }^{2}$ Participants were evaluated initially between September 1, 1999, and September 30, 2002, and reevaluated again approximately 5 years later between December 8, 2004, and July 12, 2007. Disease-specific diabetes knowledge was assessed at baseline and at follow-up by the general subscale of the Diabetes Knowledge Test $(\mathrm{DKT}),{ }^{3}$ which was administered in a 1-to-1 interview by research personnel.

The test items are designed to be representative of the larger domain of illness-specific diabetes knowledge appropriate for individuals with type 2 diabetes. The score is calculated as the percentage of correct answers out of 14 multiple choice test items that assess diabetes-related issues including dietary choices, blood glucose testing, and medical problems that are associated with diabetes. We compared the percentage correct for the total score and 
for each item on the DKT at baseline and follow-up using a paired $t$ test and McNemar $\chi^{2}$ tests, respectively. We also compared the percentage correct on the DKT between our sample and a community sample referent group of 811 adults with diabetes, almost all type $2,{ }^{3}$ using an ordinary $t$ test and Pearson $\chi^{2}$ tests.

Results. A total of 95 of the persons with serious mental illness were reevaluated at the 5-year follow-up. Sample characteristics were mean (SD) age of 49.37 (9.11) years; $\mathrm{n}=50$ (53\%) male; $\mathrm{n}=44$ (46\%) Caucasian; $\mathrm{n}=45$ (47\%) African American; $\mathrm{n}=65$ (68\%) at least high school education; mean (SD) duration of diabetes 13.2 (7.5) years. The sample was divided between those with a diagnosis of a major mood disorder $(n=50,53 \%)$ and schizophrenia $(\mathrm{n}=45,47 \%)$. At follow-up, they were receiving the following antipsychotic medications: olanzapine $(n=15,16 \%)$; clozapine $(n=3$, $3 \%)$; risperidone $(n=22,23 \%)$; quetiapine $(n=17,18 \%)$; ziprasidone $(\mathrm{n}=3,3 \%)$; aripiprazole $(\mathrm{n}=11,12 \%)$; and first-generation antipsychotic agents $(n=24,25 \%)$.

The sample of $n=95$ represents $47.3 \%$ of the original sample of $\mathrm{N}=201$ who were evaluated at baseline. Persons who could not be followed up did not differ significantly from those who were reevaluated in terms of baseline demographic variables of age, race, gender, education, psychiatric diagnosis (schizophrenia vs mood disorder), or baseline DKT score (all $P>.14$ ).

The mean (SD) score on the DKT at follow-up was 56\% (19\%). Fifty-six of the 95 persons in the sample answered at least 6 of the 14 items incorrectly. The items that were the most likely to be answered incorrectly were those concerning the diabetes diet. For example, only $36 \%(n=34)$ of the sample endorsed the correct response to the question, "What is a free food?" from among the 4 response options; the correct response was "any food that has less than 20 calories per serving." Similarly, in response to the question, "Which of the following is highest in fat?" only $24 \%$ $(n=23)$ of the sample endorsed the correct response of "low fat milk." Generally low correct response rates were also found for the items concerning blood glucose. Only $34 \%(n=32)$ of the sample correctly endorsed that the time period for which glycosylated hemoglobin (hemoglobin $\mathrm{A}_{1 \mathrm{c}}$ ) measures average blood glucose is the past 6-10 weeks rather than much longer or shorter time intervals. The percentage of correct responses was higher on items concerning diabetes-related health problems, although for no item did the overall percentage correct exceed $80 \%$. A full $80 \%(n=76)$ of the sample endorsed correctly that the correct way to take care of their feet is to "look at and wash them everyday."

The average score for persons in the sample on the DKT was not significantly changed from their baseline score. No individual item had a significant increase in mean correct responses $(P>.05)$. No baseline demographic or clinical variables predicted a change in knowledge score. A comparison of scores in our serious mental illness sample with those from a community referent group showed that the community comparison group had a significantly higher percentage correct on 8 of 14 items.

Diabetes-specific knowledge in persons with serious mental illness and co-occurring type 2 diabetes remains markedly low despite the increased attention to diabetes in this population. Persons in our sample showed a particular deficit in knowledge related to diabetes dietary issues that has implications for the ability of persons to manage their diet effectively on a day-to-day basis, a central aspect of diabetes self-care. Dietary information may less likely be a focus of interactions with medical care providers than are health-related diabetes problems that are more directly medical in content.

In the approximately 5 -year interval between the baseline and follow-up assessments of our study, there have been increased initiatives to better identify, assess, and treat persons with serious mental illness and type 2 diabetes. ${ }^{4}$ The fact that the diabetes- specific knowledge of participants did not improve during this time period is concerning. Our data suggest that there is a gap between the current focus on diabetes management of persons with serious mental illness in the medical literature and the level of information about diabetes that is acquired by patients with co-occurring serious mental illness and diabetes in routine clinical settings. Increased education and discussion about diabetes by psychiatric clinicians with their patients who have diabetes are called for.

\section{REFERENCES}

1. Dickerson FB, Goldberg RW, Brown CH, et al. Diabetes knowledge among persons with serious mental illness and type 2 diabetes. Psychosomatics. 2005;46:418-424.

2. Dixon LB, Kreyenbuhl JA, Dickerson FB, et al. A comparison of type 2 diabetes outcomes among persons with and without severe mental illnesses. Psychiatr Serv. 2004(8);55:892-900.

3. Fitzgerald JT, Funnell MM, Hess GE, et al. The reliability and validity of the brief Diabetes Knowledge Test. Diabetes Care. 1998;21(5): 706-710.

4. Sernyak MJ. Implementation of monitoring and management guidelines for second-generation antipsychotics. J Clin Psychiatry. 2007;68 (suppl 4):14-18.

Faith B. Dickerson, PhD Julie Kreyenbuhl, PharmD, PhD Richard W. Goldberg, PhD LiJuan Fang, MS Deborah Medoff, PhD Clayton H. Brown, PhD Lisa Dixon, MD

Author affiliations: Sheppard Pratt Health System (Dr Dickerson); the VA Capitol Healthcare Network Mental Illness Research, Education, and Clinical Center (Drs Kreyenbuhl, Goldberg, Brown, and Dixon); and the Departments of Psychiatry (Drs Kreyenbuhl, Goldberg, Medoff, Brown, and Dixon and Ms Fang) and Epidemiology (Dr Brown), University of Maryland School of Medicine, Baltimore, Maryland. Financial disclosure: None reported. Funding/support: The work described in this letter was supported in part by an unrestricted grant from Bristol-Meyers Squibb and by National Institutes of Health grant RO1MH058717. doi:10.4088/JCP.08l04602

(C) Copyright 2009 Physicians Postgraduate Press, Inc.

\section{Aripiprazole Treatment of Risperidone-Induced Hyperprolactinemia}

Sir: Hyperprolactinemia is a well-recognized complication of some antipsychotic agents that results from the blocking of dopamine- $2\left(\mathrm{D}_{2}\right)$ receptors in the anterior pituitary. ${ }^{1}$ Aripiprazole, a potent partial agonist of the $\mathrm{D}_{2}$ receptors, ${ }^{2}$ inhibits spontaneous prolactin release from isolated anterior pituitary slices. ${ }^{3}$ Clinically, switching to aripiprazole monotherapy resolves antipsychoticinduced hyperprolactinemia. ${ }^{4,5}$ A double-blind, placebo-controlled trial and a few single case reports demonstrated that the addition of aripiprazole reversed haloperidol-induced hyperprolactinemia and associated symptoms. ${ }^{6-8}$ We conducted an 8-week, prospective, open-label study to assess whether adjunctive treatment with aripiprazole would improve risperidone-induced hyperprolactinemia.

Method. Twenty-one male Chinese outpatients and inpatients meeting DSM-IV criteria for schizophrenia were recruited after they gave informed consent and institutional review board approval was obtained. Data were collected from December 2007 to June 2008.

Nineteen subjects completed the trial, receiving a fixed dose of $10 \mathrm{mg} / \mathrm{d}$ of aripiprazole at 7:00 AM for 8 weeks. The doses of 
risperidone and concomitant medications remained fixed throughout the study. Serum prolactin levels were measured at baseline and 2, 4, and 8 weeks using standard radioimmunoassay. Symptom severity was assessed using the Positive and Negative Syndrome Scale (PANSS) ${ }^{9}$ and the Clinical Global Impressions scale (CGI). ${ }^{10}$ Tolerability was evaluated with the Barnes Akathisia Scale (BAS) ${ }^{11}$ and the Simpson Angus Scale (SAS). ${ }^{12}$ The same investigator rated patients with these scales at baseline and at 8 weeks.

Results. Mean \pm SD prolactin levels at baseline and 2, 4, and 8 weeks were $62.4 \pm 22.1 \mathrm{ng} / \mathrm{mL}, 26.1 \pm 11.2 \mathrm{ng} / \mathrm{mL}, 18.9 \pm 8.1$ $\mathrm{ng} / \mathrm{mL}$, and $18.1 \pm 7.7 \mathrm{ng} / \mathrm{mL}$, respectively $(F=41.68, d f=5$, $P<.0001)$. Pairwise comparisons showed that prolactin levels significantly decreased between baseline and 2, 4, and 8 weeks, and between 2 and 4 weeks $(P<.0001)$, with no significant difference between 4 and 8 weeks. At the completion of the study, all patients demonstrated significantly reduced serum prolactin levels, and 6 of 19 (32\%) patients had clinically normal prolactin levels.

The patients showed no significant changes in PANSS or CGI scores or in SAS or BAS scores between baseline and week 8. Only a few side effects were noted, including tachycardia $(n=2)$ and anorexia and headache $(n=1$; both side effects were in the same patient).

To our knowledge, this is the first open-label prospective trial of aripiprazole to treat risperidone-induced hyperprolactinemia. Our study found that 8 weeks of adjunctive aripiprazole treatment was effective, safe, and well tolerated in reducing elevated prolactin levels. The mechanism for the resolution of hyperprolactinemia using aripiprazole is likely due to its unique partial agonist activity at $\mathrm{D}_{2}$ receptors and relatively high $\mathrm{D}_{2}$ receptor affinity. ${ }^{13}$ The partial agonist property means that, in the presence of dopamine (DA) hypoactivity, as induced by risperidone, aripiprazole will function as a DA agonist, restoring tonic inhibition to anterior pituitary lactotrophs, where tonic DA stimulation controls prolactin secretion. ${ }^{14,15}$

Limitations of the present study include the relatively small sample size, the short duration of the trial, and the use of the fixed dose of aripiprazole. Therefore, larger, double-blind, placebo-controlled studies of longer duration with different doses of aripiprazole (especially higher doses, such as 20 or even $30 \mathrm{mg}$ ) might further investigate efficacy, safety, tolerability, and a dosedependent effect of adjunctive aripiprazole.

\section{REFERENCES}

1. Jung DU, Seo YS, Park JH, et al. The prevalence of hyperprolactinemia after long-term haloperidol use in patients with chronic schizophrenia. J Clin Psychopharmacol. 2005;25(6):613-615.

2. Kessler RM. Aripiprazole: what is the role of dopamine D2 receptor partial agonism? Am J Psychiatry. 2007;164(9):1310-1312.

3. Inoue T, Domae M, Yamada K, et al. Effects of the novel antipsychotic agent 7-[4-[(2,3-dichlorophenyl)-1-piperazinyl] butyoxy]-3,4-dihydro-2 $\left({ }^{\mathrm{I}} \mathrm{H}\right)$-quinolinone (OPC-14597) on prolactin release from the rat anterior pituitary gland. J Pharmacol Exp Ther. 1996;277(1):137-143.

4. Mir A, Shivakumar K, Williamson RJ, et al. Change in sexual dysfunction with aripiprazole: a switching or add-on study. J Psychopharmacol. 2008;22(3):244-253.

5. Lee BH, Kim YK, Park SH. Using aripiprazole to resolve antipsychotic-induced symptomatic hyperprolactinemia: a pilot study. Prog Neuropsychopharmacol Biol Psychiatry. 2006;30(4):714-717.

6. Shim JC, Shin JG, Kelly DL, et al. Adjunctive treatment with a dopamine partial agonist, aripiprazole, for antipsychotic-induced hyperprolactinemia: a placebo-controlled trial. Am J Psychiatry. 2007;164(9):1404-1410.

7. Chen $\mathrm{CH}, \mathrm{Lu} \mathrm{ML}$. Aripiprazole resolves symptomatic hyperprolactinemia in a male schizophrenic patient. Prog Neuropsychopharmacol Biol Psychiatry. 2008;32(3):893-894.

8. Wahl R, Ostroff R. Reversal of symptomatic hyperprolactinemia by aripiprazole. Am J Psychiatry. 2005;162(8):1542-1543.

9. Kay SR, Opler LA, Fizbein A. The Positive and Negative Syndrome Scale (PANSS) Manual. North Tonawanda, NY: Multi-Health System; 1986.

10. Guy W. ECDEU Assessment Manual for Psychopharmacology. US Dept Health, Education, and Welfare publication (ADM) 76-338. Rockville, MD: National Institute of Mental Health; 1976:218-222.

11. Barnes TRE. A rating scale for drug-induced akathisia. Br J Psychiatry. 1989;154:672-676.

12. Simpson GM, Angus JWS. A rating scale for extrapyramidal side effects. Acta Psychiatr Scand Suppl. 1970;212:11-19.

13. El-Sayeh HG, Morganti C, Adams CE. Aripiprazole for schizophrenia: systematic review. Br J Psychiatry. 2006;189:102-108.

14. Grunder G, Carlsson A, Wong DF. Mechanism of new antipsychotic medications: occupancy is not just antagonism. Arch Gen Psychiatry. 2003;60(10):974-977.

15. Aihara K, Shimada J, Miwa T, et al. The novel antipsychotic aripiprazole is a partial agonist at short and long isoforms of D2 receptors linked to the regulation of adenylyl cyclase activity and prolactin release. Brain Res. 2004;1003(1-2):9-17.

Jing Xu Chen, MD

Yun Ai Su, MD, PhD

Shao Li Wang, MD

Qing Tao Bian, MD

Yan Hong Liu, MD

Ning Wang, MD

Fu De Yang, MD

Colin Haile, PhD

Thomas R. Kosten, MD

Xiang Yang Zhang, MD, PhD

Author affiliations: Beijing Hui-Long-Guan Hospital (Drs Chen, S. L. Wang, Bian, Liu, N. Wang, Yang, and Zhang) and Institute of Mental Health, Peking University (Dr Su), Beijing, China; and Department of Psychiatry, Baylor College of Medicine, Houston, Texas (Drs Haile, Kosten, and Zhang). Authorship note: Drs Chen and Su contributed equally to this work. Financial disclosure: None reported. Funding/support: This study was funded by the Stanley Medical Institute Foundation (03T-459, 05T-726) (Dr Zhang) and the Department of Veterans Affairs, VISN 1, Mental Illness Research, Education and Clinical Center (MIRECC) and National Institute on Drug Abuse K05-DA0454 and P50-DA18827 (Dr Kosten).

doi:10.4088/JCP.08l04671

(c) Copyright 2009 Physicians Postgraduate Press, Inc. 